\title{
Benzotriazole Enhances Cell Invasive Potency in Endometrial Carcinoma Through CTBP1-Mediated Epithelial- Mesenchymal Transition
}

\author{
Yiquan Wang ${ }^{\mathrm{b}}$ Chencheng Dai ${ }^{\mathrm{a}}$ Cheng Zhou ${ }^{\mathrm{c}}$ Wenqu Lia Yujia Qian ${ }^{\mathrm{a}}$ \\ Juan Wen ${ }^{\mathrm{a}}$ Yang Wang ${ }^{\mathrm{e}}$ Bing Han ${ }^{\mathrm{d}}$ Jingjing $\mathrm{Ma}^{\mathrm{a}}$ Juan $\mathrm{Xu}^{\mathrm{a}}$ Ziyi Fu \\ Hongjie Ruan ${ }^{\mathrm{a}}$ Hua Tong ${ }^{\mathrm{a}}$ Xuemei Jia ${ }^{\mathrm{a}}$
}

${ }^{a}$ Nanjing Maternal and Child Health Medical Institute, Obstetrics and Gynecology Hospital Affiliated to Nanjing Medical University, Nanjing, 'bShanghai Longhua Hospital, Affiliated to Shanghai TCM University, Shanghai, 'Department of Emergency, Nanjing First Hospital, Affiliated to Nanjing Medical University, Nanjing, 'Key Laboratory of Integrated Regulation and Resource Development on Shallow Lake of Ministry of Education, College of Environment, Hohai University, Nanjing, eDepartment of Obstetrics and Gynecology, Lianyungang First people's Hospital, Lianyungang, China

\section{Key Words}

Benzotriazole $\bullet$ Endometrial carcinoma $•$ Metastasis and invasion $\bullet$ Gene microarray $\bullet$ Epithelialmesenchymal transition

\begin{abstract}
Background/Aims: Benzotriazole (BTR) and its derivatives, such as intermediates and UV stabilizers, are important man-made organic chemicals found in everyday life that have been recently identified as environmental toxins and a threat to female reproductive health. Previous studies have shown that BTR could act as a carcinogen by mimicking estrogen. Environmental estrogen mimics could promote the initiation and development of female cancers, such as endometrial carcinoma, a type of estrogenic-sensitive malignancy. However, there is little information on the relationship between BTR and endometrial carcinoma. In this study, we aimed to demonstrate the biological function of BTR in endometrial carcinoma and explored the underlying mechanism. Methods: The CCK-8 assay was performed to detect cell viability; transwell-filter assay was used to assess cell invasion; gene microarray analysis was employed to determine gene expression patterns in response to BTR treatment; western blotting and quantitative reverse transcription polymerase chain reaction (qRT-PCR) were carried out to detect the expression levels of BTR-related genes. Results: Our data showed that BTR could induce the invasion and migration of endometrial carcinoma cells (Ishikawa and HEC-1-B). In addition, BTR increased the expression level of CTBP1, which could enhance the epithelialmesenchymal transition (EMT) in cancer cells. Moreover, CTBP1 silencing reversed the effect of

Y. Wang, C. Dai and C. Zhou contributed equally to this work.

Xuemei Jia, Hua Tong and Hongjie Ruan

Nanjing Maternal and Child Health Medical Institute, Affiliated Nanjing Maternal and Child Health Hospital,Nanjing Medical University, 123 Mochou Rd, Nanjing (China) Tel. +8625 52226159, Fax +862552226159, E-Mail xuemeijia1@sina.com
\end{abstract}

\section{KARGER}




\section{Cellular Physiology Cell Physiol Biochem 2017;44:2357-2367

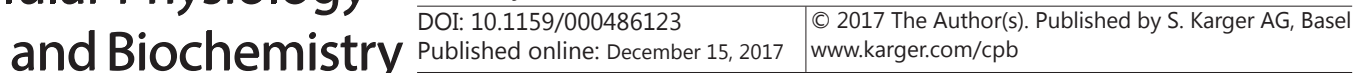 \\ Wang et al.: Benzotriazole Stimulate Endometrial Carcinoma Cells Invasion by CTBP1}

BTR on EMT progression in endometrial carcinoma cells. Conclusion: This study indicates that BTR could act as a carcinogen to promote the development of endometrial carcinoma mainly through CTBP1-mediated EMT, which deserves more attention.

\section{Introduction}

Benzotriazole (BTR) is an important intermediate of organic synthesis and a type of environmental toxin that is widely found in everyday life. BTR and its derivatives are used as anti-corrosive agents in dishwashing detergents, automotive antifreeze formulations and deicing fluids for aircrafts [1], as well as UV stabilizers in various consumer and industrial products, including wax, paint, household products, cosmetics, and sunscreens [2]. Several studies have identified the toxic effects of BTR derivatives on the human liver, heart, spleen, kidneys and thyroids ( $>12.5 \mathrm{mg} / \mathrm{kg}$ in females, $\sim 0.54 \mu \mathrm{mol} / \mathrm{L}$ ) [3], and animal blood [4]. BTR can disrupt the activity of several pathways, including estrogen, androgen and thyroid signaling [5-7]. More importantly, BTR and its derivatives have been classified as potential carcinogens and estrogen mimics [1].

Endometrial carcinoma is one of the most common malignancies of the female reproductive system. It is reported that approximately 320,000 women worldwide are diagnosed with endometrial cancer each year, and 76, 000 women die from this disease [8]. Thus, it is important to elucidate the factors influencing endometrial carcinoma development. For example, it has been demonstrated that benzene compounds, such as BTR and its derivatives, can stimulate estrogen production through the estrogen receptor [9], and as an estrogen-sensitive malignancy [8], endometrial carcinoma may be associated with BTR exposure in daily life. However, little is known about the link between BTR and endometrial carcinoma development.

During past decades, a number of studies have demonstrated that cancer cell invasion can be induced by many physicochemical factors, including extracellular calcium [10], environmental toxins [11], progesterone [12], and bacterial peptidoglycan [13], though the relationship between environmental estrogens and tumor metastasis remains unknown. Tumor metastasis is a key process during carcinoma development, and the major cause of treatment failure. Tumor metastasis occurs through a series of steps, including cellular detachment and invasion [14]. Prior to invasion, cancer cells need to acquire migratory and invasive properties via the epithelial-mesenchymal transition (EMT) [15]. The key steps of EMT include the loss of cell polarity and acquisition of mesenchymal phenotype, which are accompanied by reduced expression of the epithelial marker E-cadherin and increased expression of mesenchymal markers, such as N-cadherin and Vimentin [16]. EMT-associated transcription factors, such as Snail and CTBP1, are known to decrease E-cadherin expression, thereby inducing EMT $[17,18]$. EMT enables tumor cells to invade through the local matrix and into blood vessels, ultimately resulting in distant metastasis to other organs.

In this study, we aimed to reveal the possible effects of BTR on endometrial carcinoma cell lines and the underlying mechanisms. In addition, our findings raise awareness regarding the side effects of everyday products that contain BTR and its derivatives. Furthermore, we sought to translate the findings from basic research to the clinical treatment of endometrial carcinoma caused by environmental estrogen mimics such as BTR.

\section{Materials and Methods}

\section{Reagents}

BTR (purity $\geq 95 \%$, by HPLC) was purchased from Sigma (USA). Ishikawa and HEC-1-B endometrial carcinoma cell lines were obtained from the American Type Culture Collection. Heat-inactivated fetal bovine serum (FBS) and cell culture medium DMEM was obtained from Gibco (Invitrogen China Limited, China). Cell culture medium RPMI-1640 was purchased from Wisent (China). Other reagents were purchased from Sigma-Aldrich Inc. unless otherwise mentioned. 


\section{Cellular Physiology Cell Physiol Biochem 2017;44:2357-2367 \begin{tabular}{l|l|l} 
DOI: 10.1159/000486123 & $\begin{array}{l}\text { O 2017 The Author(s). Published by S. Karger AG, Basel } \\
\text { www.karger.com/cpb }\end{array}$
\end{tabular}}

\section{Cell culture and BTR exposure}

Ishikawa endometrial carcinoma cells were maintained in RPMI-1640 medium (containing 100U/ $\mathrm{ml}$ streptomycin, 100U/ml penicillin, 10\% FBS). HEC-1-B endometrial carcinoma cells were maintained in DMEM medium (containing $100 \mathrm{U} / \mathrm{ml}$ streptomycin, $100 \mathrm{U} / \mathrm{ml}$ penicillin, 10\% FBS) [19]. Cells were cultured in a humidified atmosphere of $5 \%$ carbondioxide at $37^{\circ} \mathrm{C}$. BTR was dissolved in RPMI- 1640 and DMEM medium separately to make a $200 \mathrm{mM}$ stock solution and added to the media to reach different doses $(0,1,10,100,1000 \mathrm{nM})$.

\section{Cell Counting Kit-8 (CCK8) assay}

Cell viability was calculated by Cell Counting Kit-8 (CCK8) assay according to the manufacturer's protocol [20]. Ishikawa and HEC-1-B cells were seeded into a 96-well plate and then cultured with serumfree medium (Ishikawa 2000 cells/well, HEC-1-B 1600 cells/well). $24 \mathrm{~h}$ later, the cells were treated with different doses of BTR $(0,1,10,100,1000 \mathrm{nM})$. After $72 \mathrm{~h}$ treatment, the cells were incubated with $10 \mu \mathrm{l}$ CCK8/well dissolved in growth medium at $3^{\circ} \mathrm{C}$ for $2 \mathrm{~h}$. The absorbance of each well was measured at $450 \mathrm{~nm}$ by Synergy 2 Absorbance Microplate Hybird Reader (Biotek, USA). Cell viability was expressed as a fraction of absorbance compared to control.

\section{Trans-well filter assay}

The invasion assay in vitro was carried out using transwell chamber with $8 \mathrm{~mm}$ diameter pore size polycarbonate membrane (Corning Costar, Cambridge, Massachusetts) coated with $20 \mu \mathrm{g}$ matrigel as described previously [21]. Ishikawa and HEC-1-B cells were trypsinized separately and added into each upper chamber of transwell $\left(8^{*} 10^{4}\right.$ cells/well). The lower chamber was filled with culture medium, containing 10\% FBS. After cell adhered to dish, culture medium was replaced by serum-free media with different doses of BTR $(0,1,10,100 \mathrm{nM}) .24 \mathrm{~h}$ later, the invaded cells were fixed with $4 \%$ Paraformaldehyde and then stained with crystal violet staining solution (Millipore, Billerica). The invaded cells were dissolved in lysis buffer, and OD volume of each group was detected by microplate reader (Biotek, USA).

\section{Total RNA extraction}

Ishikawa cells were dissolved in TRIzol reagent and total RNAs were extracted according to the manufacturer's protocol (Invitrogen, CA, USA) [22]. Quantification and quality checks were performed with Nano-drop and an Agilent 2100 Bio-analyzer (Agilent Technologies) respectively.

\section{Gene microarray analysis}

Global gene expression was detected using Affyme-trix GeneChip Human Gene 1.0 ST Array (Affymetrix, USA) [23]. About $500 \mathrm{ng}$ of total RNA were employed in each experiment. Briefly, total RNA of each sample was amplified using the Ambion WT Expression Kit (Applied Biosystems, Foster City, USA) according to the manufacturer's instructions. Approximately $5.5 \mathrm{mg}$ of generated cDNA was fragmented and labeled using GeneChip3 WT Terminal Labeling Kit and Controls Kit (Affymetrix, USA). Labeled cDNA target was hybridized to the Affymetrix GeneChip Human Gene 1.0 ST Array using GeneChip3 Hybridization, Wash, and Stain Kit (Affymetrix, USA) at $45^{\circ} \mathrm{C}$ for $16 \mathrm{~h}$, then hybridized arrays were washed and stained on the Affymetrix GeneChip Command Console (AGCC, Affymetrix, USA), than scanned on a GeneChip2 Scanner 3000 7G ((Affymetrix, USA). The acquired array images were analyzed by Affymetrix GeneChip Operating Software (GCOS). Affymetrix expression console software was used to perform quantile normalization and subsequent data processing. Data transformation was applied to set all negative raw values at 1.0, followed by quantile normalization. A filter on low gene expression was used to keep only the probes expressed in at least one sample. Differentially regulated genes were identified via fold change filtering.

\section{Gene ontology (GO) and Kyoto encyclopedia of genes and genomes (KEGG) analysis}

GO function and KEGG enrichment for differentially expressed genes were used to identify the significantly enriched biological terms and pathways [24]. The database for annotation, visualization, and integrated discover (DAVID) was applied to perform GO function enrichment for differentially expressed genes. Putative genes that were up/down-regulated more than 2-fold with a P value less than 0.05 after treated with BTR compared to control group were considered as differentially expressed and selected to submit to David Bioinformatics Resources 6.7, NIAID/NIH (http://david.abcc.ncifcrf.gov/). The overall 


\section{Cellular Physiology Cell Physiol Biochem 2017;44:2357-2367 \begin{tabular}{l|l|l} 
and Biochemistry Published online: December 15, 2017 & $\begin{array}{l}\text { (c) } 2017 \text { The Author(s). Published by S. Karger AG, Basel } \\
\text { www.karger.com/cpb }\end{array}$
\end{tabular}}

functions regulated by BTR were identified by functional annotation clustering and ranked by enrichment scores.

\section{Quantity Real-time PCR}

The total RNA of sample tissues and cells was extracted and reverse transcribed into cDNA with random primers with a Reverse Transcription Kit (Takara) according to the manufacturer's instructions. Standard qRT-PCR was performed to confirm the expression levels of differentially expressed lncRNAs with the Applied Biosystems ViiA 7 Sequence Detection System (ABI ViiA 7 SDS, USA) following the manufacturer's guidelines [25]. Briefly, the mixture of samples was incubated at $95{ }^{\circ} \mathrm{C}$ for $10 \mathrm{~min}$ for an initial denaturation, followed by 40 PCR cycles of incubation at $95{ }^{\circ} \mathrm{C}$ for $15 \mathrm{~s}, 60{ }^{\circ} \mathrm{C}$ for $30 \mathrm{~s}$, and then $72{ }^{\circ} \mathrm{C}$ for $30 \mathrm{~s}$. The specific primer sequences for qRTPCR were: N-cadherin, forward, 5'-GAGATCCTACTGGA CGGTTCG-3' and reverse, 5'-TCTTGGCGAATGATCTTAGGA-3'; Vimentin, forward, 5'-CCTTGAACGCAA-AGTGGAATC-3' and reverse, 5'-TGAGGTCAGG CTTGGAAACAT-3'; E-cadherin, forward, 5'-GACCG-AGAGAGTTTCCCTACG-3' and reverse, 5'-TCAGGCACCTGACCCTTGTA-3'; CtBP1, forward, 5'-CCATCA CTCTCACCAGGGA-3' and reverse, 5'-GCCGTCTCCTC CACAGA-30;GAPDH, forward, 5'-GGTCTC CTCTGACTTCAACA-3' and reverse, 5'-AGCCAAATTCGT TGTCATAC-3'. Each sample analysis was performed in triplicate. The expression levels of IncRNAs were normalized to internal control GAPDH, and then calculated with the 2- $\Delta$ CT method.

\section{Western blotting analysis}

Ishikawa cells were lysed by RIPA buffer (Thermo Fisher, USA), a standard Western blotting analysis was conducted as previously described to investigate the expression level of CTBP1, E-cadherin, N-cadherin, and Vimentin [26]. Briefly, protein lysates were boiled in sodium dodecyl sulfate (SDS)-sample buffer for 5 min before SDSpolyacrylamide gel electrophoresis (8\%) and transfer to polyvinylidene fluoride membranes (Bio-Rad). Membranes were blocked for $2 \mathrm{~h}$ in 5\% milk-Tris-Buffered Saline Tween-20 (TBST) at room temperature and incubated with the monoclonal antibodies (Cell Signaling Technology). Membranes were washed four times in TBST and incubated with the appropriate horseradish peroxidase-conjugated secondary antibodies. Blots were visualized by enhanced chemiluminescence (Thermo) and analyzed using a scanning densitometer of Gel Imaging System (Protein Simple, USA).

\section{Statistics}

Experimental results were statistically analyzed using SPSS 11.0 (SPSS Inc., Chicago, USA). All values were expressed as mean standard deviation (SD). The significance of the difference between the control and the BTR groups was assessed by applying one way ANOVA (analysis of variance) method followed by post hoc Tukey's test using SPSS, and $p<0.05$ was considered statistically significant.

\section{Results}

Effect of BTR on the invasive ability of endometrial carcinoma cells

Compared to control cells, 1000 nM BTR increased the viability of Ishikawa cells $(p<0.05)$ (Fig. $1 \mathrm{~A})$, whereas, no obvious change was observed in HEC-1-B cells $(p>0.05)$ (Fig. 1B). To evaluate the effect of BTR on the invasive ability of endometrial carcinoma cells, we selected certain doses BTR $(0,1,10$,

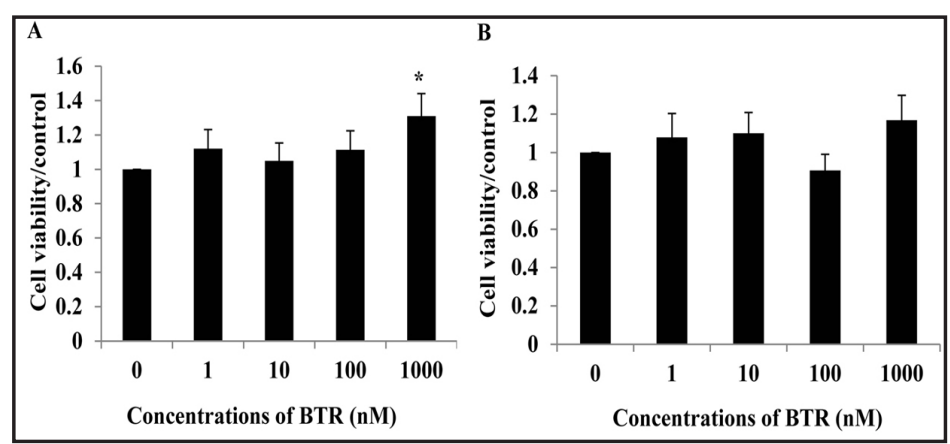

Fig. 1. BTR promoted endometrial carcinoma cells migration activity. Ishikawa and HEC-1-B cells were treated with different doses of BTR $(0,1,10,100,1000 \mathrm{nM})$ for CCK8 assay first. The data showed that $1000 \mathrm{nM}$ BTR increased cell viability of Ishikawa $(\mathrm{p}<0.05)$, but no cell viability promotion was observed in the other 3 groups $(p>0.05)$, compare to control group (A). As for HEC-1-B cells, there is no obvious change detected in all treated-groups $(p>0.05)(B)$. (Each experiment was performed at least triple times independently.). 
and $100 \mathrm{nM}$ ) for transwell assays. As shown in Fig. 2, BTR stimulated the invasive ability of Ishikawa and HEC-1-B cells (Fig. 2A) in a dose-dependent manner $\left(\mathrm{R}^{2}=0.9841\right.$ and 0.9653, respectively) as shown by crystal violet staining and assessment of OD values of the invaded cells (Fig. 2B).

Differentially expressed genes in BTR-treated cells

We detected the expression levels of genes using high-throughput microarrays. Compared to the control groups, 43 up-regulated and 35 down-regulated genes were observed after BTR treatment (Fig. 3A), including MMPs and CTBP1 (absolute fold-change $>2.0$, $p<0.05$, and FDR $<0.05$ ). To explore the potential functions of these genes

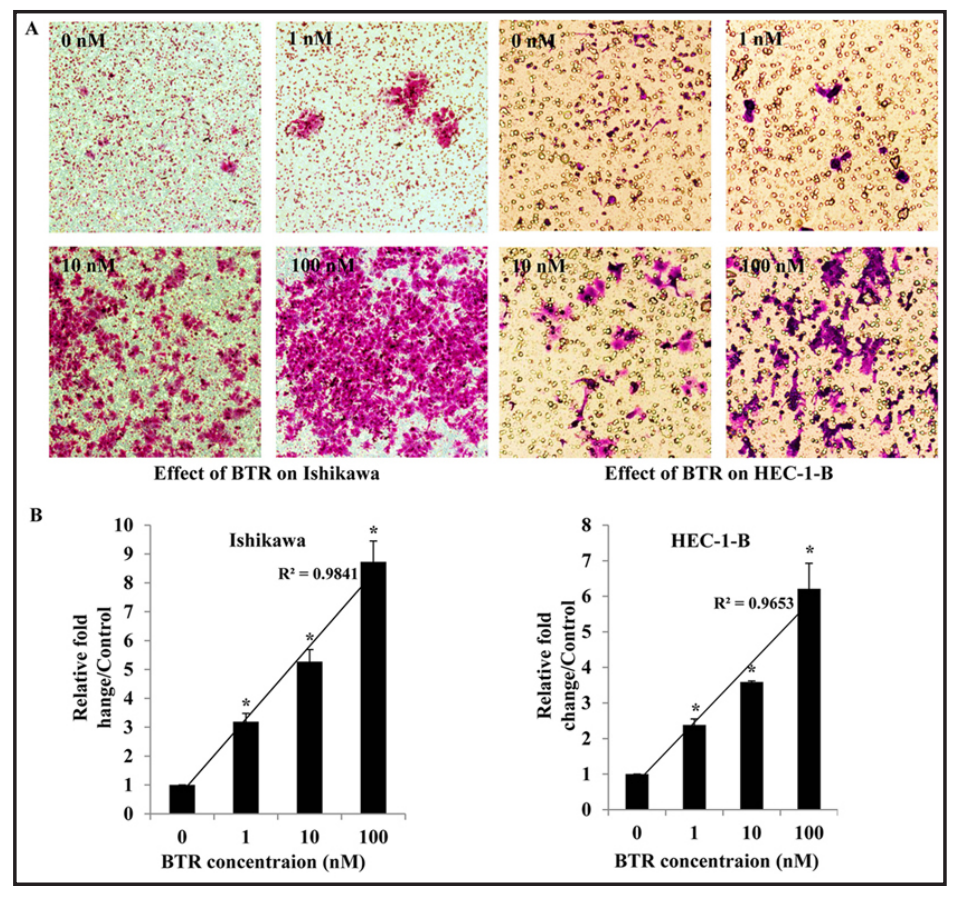

Fig. 2. BTR stimulated the invasive ability of endometrial carcinoma cells. The invasive ability of Ishikawa and HEC-1-B cells were strengthened with BTR exposure in a dose-dependent manner (A), which was confirmed by calculating the invaded cells (crystal violet stained cancer cells) (B). (Each experiment was performed at least triple times independently.). in endometrial carcinoma and their correlation with BTR, we carried out GO analysis, which indicated that these gene products are mainly involved in biological processes such as regulation of cell transition, cellular motion, and matrix degradation (Fig. 3B). In addition, KEGG pathway analysis showed that these gene products participate in several signaling pathways and processes, including focal adhesion (hsa04510), cytokine-cytokine receptor interaction (hsa04060), cell cycle (hsa04110), regulation of actin cytoskeleton (hsa04810), p53 signaling (hsa04115), adherens junction (hsa04520), Wnt signaling (hsa04310), MAPK signaling (hsa04010) and tight junction (hsa04530) (Fig. 3C). The $p$-value obtained (EASE-score, Fisher's $p$ value or Hypergeometric $p$ value) demonstrated the significance of the GO term enrichment and pathways analyzed.

\section{BTR induces EMT in endometrial carcinoma cells}

EMT, an important process during invasion and migration in various kinds of cancers. It is characterized by an increase in $\mathrm{N}$-cadherin and Vimentin expression and a reduction in E-cadherin expression. We determined the effect of BTR on EMT in the Ishikawa and HEC1-B endometrial carcinoma cell lines. Western blotting showed that BTR treatment induced expression of the mesenchymal markers $\mathrm{N}$-cadherin and Vimentin, while simultaneously decreasing that of the epithelial marker E-cadherin (Fig. 4A). Consistently, qRT-PCR results demonstrated that expression of E-cadherin mRNA was decreased and the level of Vimentin or N-cadherin mRNA was increased after BTR treatment for 24 h (Fig. 4B). These results indicated that BTR could induce EMT in endometrial carcinoma cells.

\section{BTR induces EMT through CTBP1 in endometrial carcinoma cells}

Fig. 3A shows that CTBP1, a well-recognized EMT regulator [18], was up-regulated in endometrial carcinoma cells following BTR treatment. To confirm whether CTBP1 is 


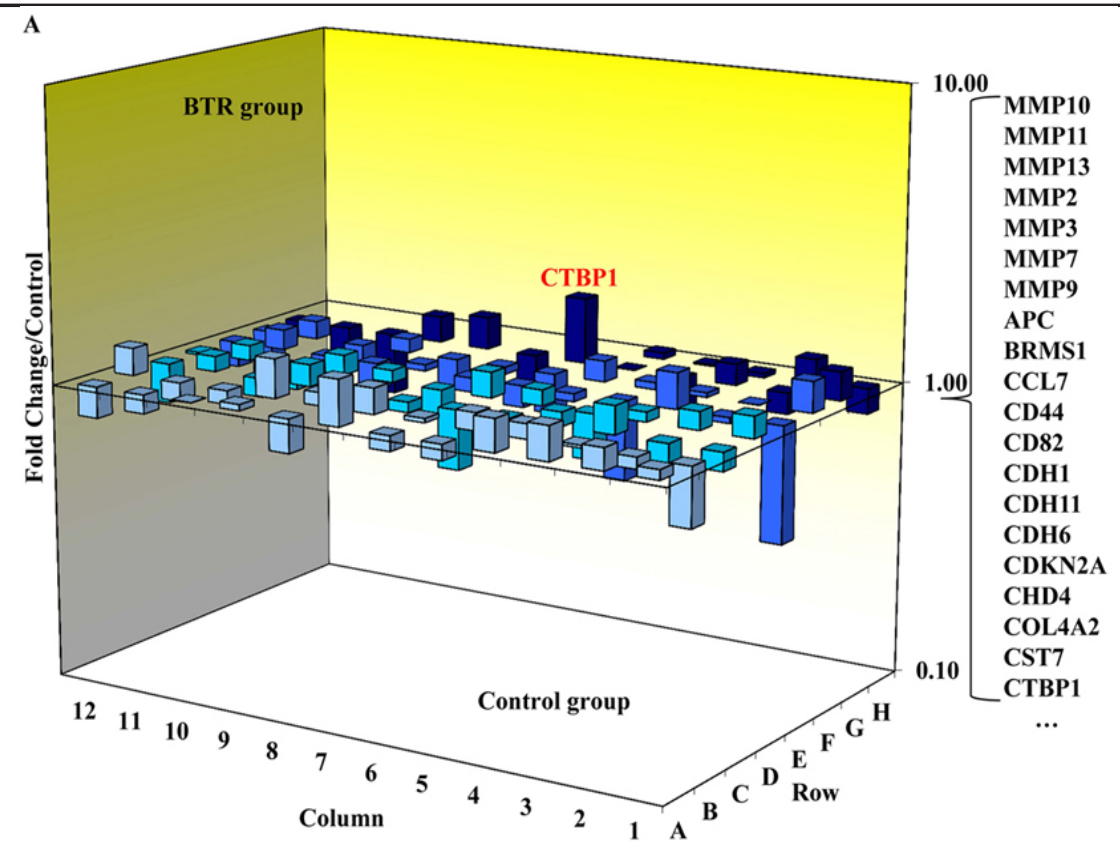

B

Sig GO terms of BP

regulation of matrix degradation collagen catabolic process cell motion negative regulation of cell proliferation multicellular organismal catabolic process collagen metabolic process behavior regulation of locomotion multicellular metabolic process regulation of cell transition

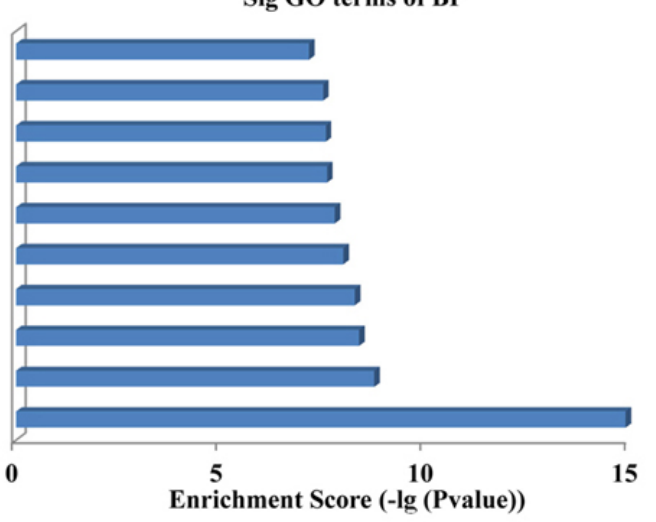

C

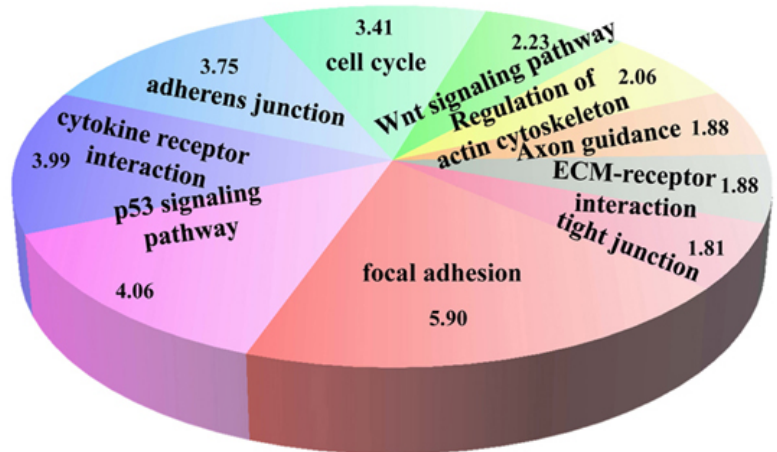

Sig pathway of DE IncRNA

EnrichmentScore (- $\lg ($ Pvalue $))$

Fig. 3. Differential genes expression characters between BTR and control group. Compared to the control group, there were 43 genes up-regulated and 35 down-regulated (A). The GO analysis results indicated that the top 10 participating biological processes of targeted genes were regulation of cell transition, multicellular metabolic process, regulation of locomotion, etc (B). The Pathway analysis results indicated that these genes were mainly involved in the focal adhesion, regulation of actin cytoskeleton, p53 signaling pathway, adherens junction, Wnt signaling pathway, and so on (C). 


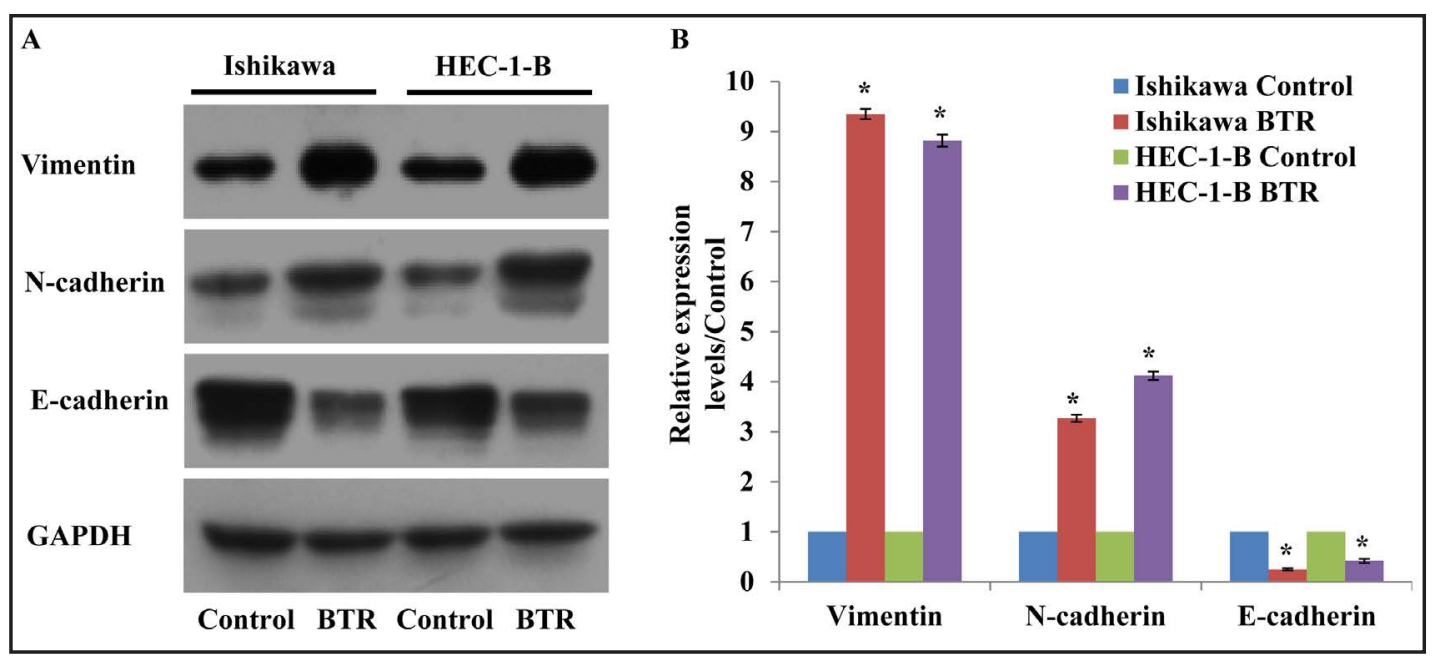

Fig. 4. BTR induces EMT in endometrial carcinoma cells. BTR induced the expression of mesenchymal markers: N-cadherin and Vimentin, while decreased the expression of epithelial marker E-cadherin concomitantly (A). Consistently, qRT-PCR results demonstrated that expression of E-cadherin mRNA was decreased and the level of Vimentin or N-cadherin mRNA was increased after BTR treatment for $24 \mathrm{~h}$ (B). (Each experiment was performed at least triple times independently.).

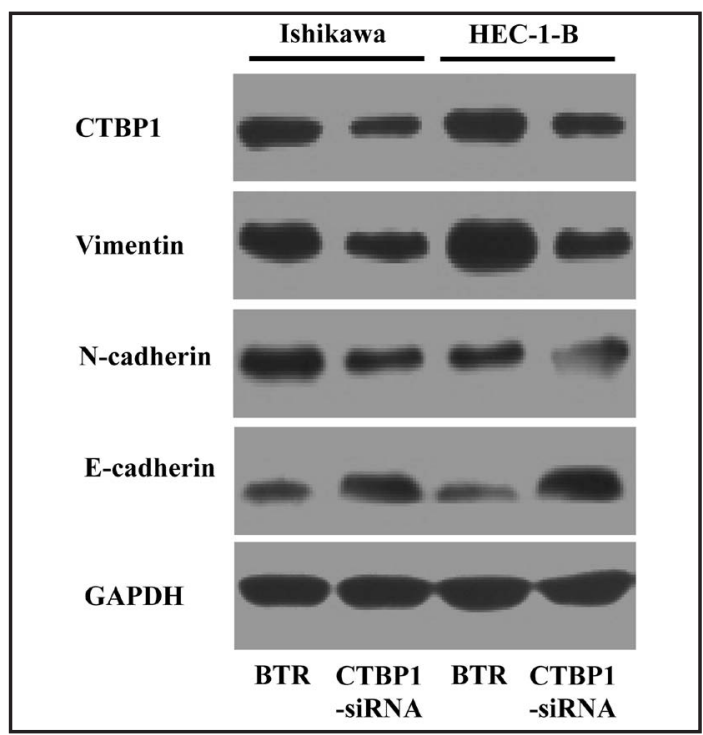

Fig. 5. BTR induces EMT through CTBP1 in endometrial carcinoma cells. BTR-induced overexpression of N-cadherin/Vimentin, and down-regulation of E-cadherin were reversed by CTBP1 siRNA both in Ishikawa and HEC-1-B cells.

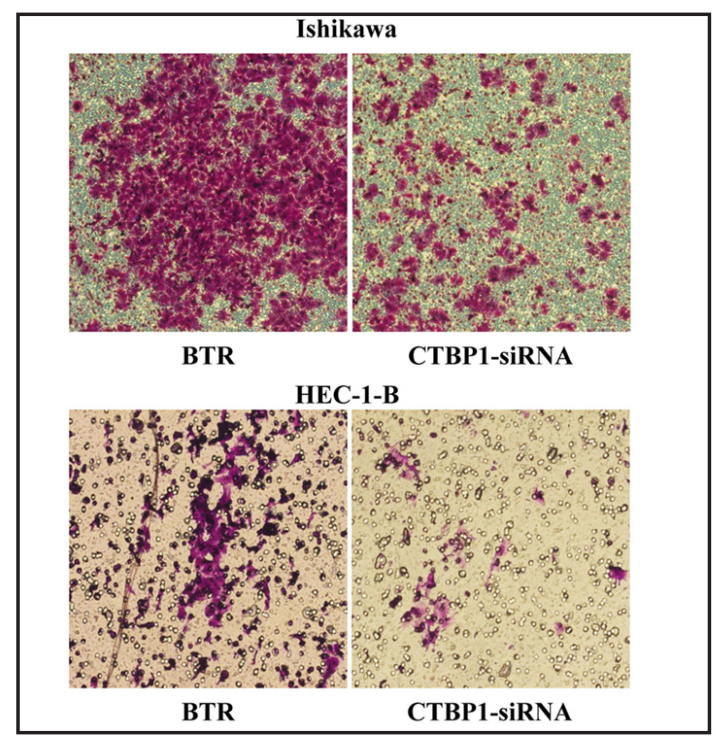

Fig. 6. BTR induces endometrial carcinoma cells' invasion through CTBP1. Induced invasive ability of endometrial carcinoma cells by BTR was blocked after transient transfection of CTBP1-siRNA.

involved in BTR-induced EMT, we suppressed endogenous CTBP1 levels using CTBP1-siRNA [27]. Western blotting showed that the expression level of CTBP1 was significantly inhibited by CTBP1-siRNA, and the BTR-induced up-regulation of N-cadherin/Vimentin and downregulation of E-cadherin were reversed by CTBP1-siRNA (Fig. 5) in both Ishikawa and HEC1-B cells. Thus, RNA interference demonstrated that CTBP1 expression contributes to BTRinduced EMT. 


\section{Cellular Physiology Cell Physiol Biochem 2017;44:2357-2367

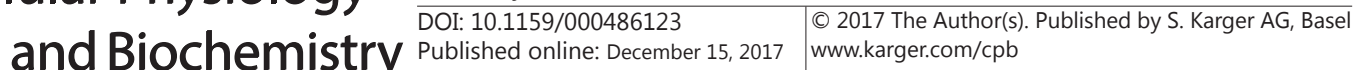 \\ Wang et al.: Benzotriazole Stimulate Endometrial Carcinoma Cells Invasion by CTBP1}

Role of CTBP1 in BTR-induced invasion of endometrial carcinoma cells

Transwell-filter assay was performed to identify the role of CTBP1 in BTR-induced cell invasion. The results showed that the BTR-induced invasive ability of endometrial carcinoma cells was blocked by transient transfection of CTBP1-siRNA (Fig. 6), thus confirming that CTBP1-siRNA suppresses the BTR-induced cellular invasiveness. Taken together, these findings indicate that overexpression of CTBP1 mediates the effect of BTR on the invasion capacity of endometrial carcinoma cells.

\section{Discussion}

Environmental toxins, such as benzene compounds, are closely associated with high rates of morbidity due to malignancies [28]. Many benzene compounds have been found to influence estrogen, androgen and thyroid signaling activities in marine organisms and mammals. However, the correlation between BTR and endometrial carcinoma, a kind of estrogen-sensitive malignancy, remains unclear. To elucidate the effect of BTR on endometrial carcinoma, we performed a CCK-8 assay. We found that the proliferation rate of both endometrial carcinoma cell lines was unaltered after exposure to low concentrations of BTR $(<1000 \mathrm{nM})$. However, low dose of BTR did enhance the invasive ability of endometrial carcinoma cells at very low doses (Fig. 2). Similar to many reported toxins, including MCLR [29] and PFOA [11], BTR was found to increase the invasive properties of cancer cells at extremely low concentrations, without affecting their proliferative abilities.

To determine the potential mechanisms of BTR-induced invasion in endometrial carcinoma cells, we assessed gene expression profiles of these cells through microarray analysis. The data showed 78 invasion-related genes to be dys-regulated in BTR-treated endometrial carcinoma cells, including MMP family members (such as MMP-2, MMP-9, and MMP-13), an EMT marker (CTBP1) and collagen (COL4A2), which are important in regulating cancer cell invasion [30]. To identify the most relevant targets, we performed GO analysis on these dysregulated genes. The results showed that these genes correspond to biological processes such as regulation of cell transition, regulation of locomotion, collagen metabolism, regulation of matrix degradation, as listed in Fig. 3B. Cell transition (such as EMT) and matrix degradation processes are both important steps in cancer invasion [30]. For example, Bouris et al. demonstrated that estrogen can induce EMT in breast cancer cells [31]. In the present study, we found that among all the regulated biological processes, the estrogen mimic BTR predominantly induced cellular transition, indicating that BTR might activate cellular invasion by inducing EMT in endometrial carcinoma cells similar to the effect of estrogen on breast cancer cells. Moreover, pathway analysis showed that focal adhesion, Wnt signaling, regulation of actin cytoskeleton, p53 signaling, and ECM-receptor interaction, which are all well-known invasion-related pathways, are among signaling pathways regulated by BTR. The correlation between focal adhesion signaling and cancer invasion has been established, and many focal adhesion proteins, including FAK, integrin, Vimentin, $\mathrm{N}$-cadherin, and E-cadherin, have been found to participate in regulating cancer cell adhesion [30]. Interestingly, one of the major steps during EMT in cancer cells is a change in cellular focal adhesion. Indeed, EMT-associated migration and metastasis in cancer cells is induced by the disruption of focal adhesion [32]. The Wnt signaling pathway has also been identified as playing a key role in lung cancer invasion [33]. Among the 78 genes altered by BTR, CTBP1, a well-recognized EMT regulator [18], has been identified in both cell transition processes and the Wnt signaling pathway. CTBP1 is an important member of the carboxyl terminal-binding protein (CTBP) family, which antagonizes the epithelial phenotype [34] by reducing expression of several epithelial genes. Thus, CTBP1 has been widely reported to have an important role in EMT regulation, indicating that the BTR-induced invasion might be related to CTBP1-mediated EMT in endometrial carcinoma cells.

EMT is also associated with the conversion of early-stage tumors into invasive malignancies, and previous studies have shown that EMT can promote cancer stemness and 


\section{Cellular Physiology Cell Physiol Biochem 2017;44:2357-2367 \begin{tabular}{l|l|l} 
DOI: 10.1159/000486123 & $\begin{array}{l}\text { O 2017 The Author(s). Published by S. Karger AG, Basel } \\
\text { www.karger.com/cpb }\end{array}$
\end{tabular}}

stimulate tumor progression in various kinds of human cancers. Additionally, EMT is believed to be an important step in cancer metastasis [35]. During the progression of malignancies, EMT causes cancer cells to lose pithelial adherence, undergo cytoskeleton remodeling, detach from the primary site, and migrate to distant sites. In this study, we found that BTR increased expression of CTBP1 and regulated that of EMT markers including N-cadherin, vimentin, and E-cadherin (Fig. 4), confirming that BTR enhances cellular invasion by inducing EMT in endometrial carcinoma cells. Additionally, a reduction of endogenous CTBP1 expression reversed the cellular invasion and EMT progression induced by BTR. Our findings demonstrate the effect of BTR on invasion and EMT in endometrial carcinoma cells and expand our knowledge regarding the pathways that participate in these cellular processes.

\section{Conclusion}

Our results showed that BTR could induce EMT, which may contribute to endometrial carcinoma metastasis. Additionally, BTR might induce EMT by up-regulating CTBP1. Therefore, our findings indicate the importance of assessing a new public health risk (e.g., the promotion of tumor metastasis) due to this toxin present in everyday products.

\section{Acknowledgements}

This study was financially supported by the National Natural Science Foundation of China (81402139, 81572556), Nanjing Municipal Science and Technology Foundation (201208025, 201402023), and The Project of Invigorating Health Care through Science, Technology and Education (Jiangsu Provincial Medical Youth Talent, QNRC2016095)

\section{Disclosure Statement}

The authors declare that they have no competing interests.

\section{References}

1 Careghini A, Mastorgio AF, Saponaro S, Sezenna E: Bisphenol A, nonylphenols, benzophenones, and benzotriazoles in soils, groundwater, surface water, sediments, and food: a review. Environ Sci Pollut Res Int 2015;22:5711-5741.

2 Nakata H, Murata S, Filatreau J: Occurrence and concentrations of benzotriazole UV stabilizers in marine organisms and sediments from the Ariake Sea, Japan. Environ Sci Technol 2009;43:6920-6926.

-3 Hirata-Koizumi M, Watari N, Mukai D, Imai T, Hirose A, Kamata E, Ema M: A 28-day repeated dose toxicity study of ultraviolet absorber 2-(2'-hydroxy-3',5'-di-tert-butylphenyl) benzotriazole in rats. Drug Chem Toxicol 2007;30:327-341.

4 Hirata-Koizumi M, Ogata H, Imai T, Hirose A, Kamata E, Ema M: A 52-week repeated dose toxicity study of ultraviolet absorber 2-(2'-hydroxy-3',5'-di-tert-butylphenyl)benzotriazole in rats. Drug Chem Toxicol 2008;31:81-96.

-5 Fent K, Chew G, Li J, Gomez E: Benzotriazole UV-stabilizers and benzotriazole: Antiandrogenic activity in vitro and activation of aryl hydrocarbon receptor pathway in zebrafish eleuthero-embryos. Sci Total Environ 2014;482-483:125-136.

-6 Kunz Y, Fent K: Estrogenic activity of ternary UV filter mixtures in fish (Pimephales promelas) - an analysis with nonlinear isobolograms. Toxicol Appl Pharmacol 2009;234:77-88. 


\section{Cellular Physiology Cell Physiol Biochem 2017;44:2357-2367

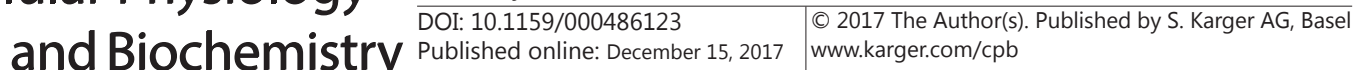

7 Schmutzler C, Bacinski A, Gotthardt I, Huhne K, Ambrugger P, Klammer H, Schlecht C, Hoang-Vu C, Grüters A, Wuttke W, Jarry H, Köhrle J: The ultraviolet filter benzophenone 2 interferes with the thyroid hormone axis in rats and is a potent in vitro inhibitor of human recombinant thyroid peroxidase. Endocrinology 2007;148:2835-2844.

-8 Murali R, Soslow RA, Weigelt B: Classification of endometrial carcinoma: more than two types. Lancet Oncol 2014;15:e268-278.

-9unz Y, Fent K: Multiple hormonal activities of UV filters and comparison of in vivo and in vitro estrogenic activity of ethyl-4-aminobenzoate in fish. Aquat Toxicol 2006;79:305-324.

10 Déliot N, Constantin B: Plasma membrane calcium channels in cancer: Alterations and consequences for cell proliferation and migration. Biochim Biophys Acta 2015;1848:2512-2522.

11 Zhang W, Wang F, Xu P, Miao C, Zeng X, Cui X, Lu C, Xie H, Yin H, Chen F, Ma J, Gao S, Fu Z: Perfluorooctanoic acid stimulates breast cancer cells invasion and up-regulates matrix metalloproteinase-2/-9 expression mediated by activating NF-kappaB. Toxicol Lett 2014;229:118-125.

12 Henriquez S, Calderon C, Quezada M, Oliva B, Bravo ML, Aranda E, Kato S, Cuello MA, Gutiérrez J, Quest AF, Owen GI: Progesterone utilizes distinct membrane pools of tissue factor to increase coagulation and invasion and these effects are inhibited by TFPI. J Cell Physiol 2011;226:3278-3285.

13 Suarez G, Romero-Gallo J, Piazuelo MB, Wang G, Maier RJ, Forsberg LS, Azadi P, Gomez MA, Correa P, Peek RM Jr: Modification of Helicobacter pylori Peptidoglycan Enhances NOD1 Activation and Promotes Cancer of the Stomach. Cancer Res 2015;75:1749-1759.

14 Carpinteiro A, Beckmann N, Seitz A, Hessler G, Wilker B, Soddemann M, Helfrich I, Edelmann B, Gulbins E, Becker KA: Role of Acid Sphingomyelinase-Induced Signaling in Melanoma Cells for Hematogenous Tumor Metastasis. Cell Physiol Biochem 2016;38:1-14.

15 Nieto MA, Huang RY, Jackson RA, Thiery JP: EMT: 2016. Cell 2016;166:21-45.

-16 Cai M, Wang Z, Zhang J, Zhou H, Jin L, Bai R, Weng Y: Adam17, a Target of Mir-326, Promotes Emt-Induced Cells Invasion in Lung Adenocarcinoma. Cell Physiol Biochem 2015;36:1175-1185.

17 Guo J, Xia N, Yang L, Zhou S, Zhang Q, Qiao Y, Liu Z: GSK-3beta and vitamin D receptor are involved in betacatenin and snail signaling in high glucose-induced epithelial-mesenchymal transition of mouse podocytes. Cell Physiol Biochem 2014;33:1087-1096.

18 Han Y, Bi Y, Bi H, Diao C, Zhang G, Cheng K, Yang Z: miR-137 suppresses the invasion and procedure of EMT of human breast cancer cell line MCF-7 through targeting CtBP1 Hum Cell 2016;29:30-36.

19 Zeng N, Salker MS, Zhang S, Singh Y, Shi B, Stournaras C, Lang F: 1alpha,25(OH)2D3 Induces Actin Depolymerization in Endometrial Carcinoma Cells by Targeting RAC1 and PAK1 Cell Physiol Biochem 2016;40:1455-1464.

20 Guo Y, Liao Y, Jia C, Ren J, Wang J, Li T: MicroRNA-182 promotes tumor cell growth by targeting transcription elongation factor A-like 7 in endometrial carcinoma. Cell Physiol Biochem 2013;32:581-590.

-21 Shi C, Zhang N, Feng Y, Cao J, Chen X, Liu B: Aspirin Inhibits IKK-beta-mediated Prostate Cancer Cell Invasion by Targeting Matrix Metalloproteinase- 9 and Urokinase-Type Plasminogen Activator. Cell Physiol Biochem 2017;41:1313-1324.

$22 \mathrm{Lv}$ J, Xia K, Xu P, Sun E, Ma J, Gao S, Zhou Q, Zhang M, Wang F, Chen F, Zhou P, Fu Z, Xie H: miRNA expression patterns in chemoresistant breast cancer tissues. Biomed Pharmacother 2014;68:935-942.

23 Lv J, Fu Z, Shi M, Xia K, Ji C, Xu P, Lv M, Pan B, Dai L, Xie H: Systematic analysis of gene expression pattern in has-miR-760 overexpressed resistance of the MCF-7 human breast cancer cell to doxorubicin. Biomed Pharmacother 2015;69:162-169.

24 Lv M, Xu P, Wu Y, Huang L, Li W, Lv S, Wu X, Zeng X, Shen R, Jia X, Yin Y, Gu Y, Yuan H, Xie H, Fu Z: LncRNAs as new biomarkers to differentiate triple negative breast cancer from non-triple negative breast cancer. Oncotarget 2016;7:13047-13059.

25 Wang H, Fu Z, Dai C, Cao J, Liu X, Xu J, Lv M, Gu Y, Zhang J, Hua X, Jia G, Xu S, Jia X, Xu P: LncRNAs expression profiling in normal ovary, benign ovarian cyst and malignant epithelial ovarian cancer. Sci Rep 2016;6:38983.

-26 Lu X, Yan C, Huang Y, Shi D, Fu Z, Qiu J, Yin Y: Mouse double minute 2 (MDM2) upregulates Snail expression and induces epithelial-to-mesenchymal transition in breast cancer cells in vitro and in vivo. Oncotarget 2016;7:37177-37191.

27 Wang R, Asangani IA, Chakravarthi BV, Ateeq B, Lonigro RJ, Cao Q Mani RS, Camacho DF, McGregor N, Schumann TE, Jing X, Menawat R, Tomlins SA, Zheng H, Otte AP, Mehra R, Siddiqui J, Dhanasekaran 


\section{Cellular Physiology Cell Physiol Biochem 2017;44:2357-2367

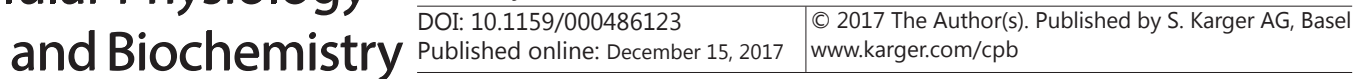 \\ Wang et al.: Benzotriazole Stimulate Endometrial Carcinoma Cells Invasion by CTBP1}

SM, Nyati MK, Pienta KJ, Palanisamy N, Kunju LP, Rubin MA, Chinnaiyan AM, Varambally S: Role of transcriptional corepressor CtBP1 in prostate cancer progression. Neoplasia 2012;14:905-914.

28 Bulka C, Nastoupil LJ, McClellan W, Ambinder A, Phillips A, Ward K, Bayakly AR, Switchenko JM, Waller L, Flowers CR: Residence proximity to benzene release sites is associated with increased incidence of nonHodgkin lymphoma. Cancer 2013;119:3309-3317.

29 Ren Y, Yang M, Chen M, Zhu Q Zhou L, Qin W, Wang T: Microcystin-LR promotes epithelial-mesenchymal transition in colorectal cancer cells through PI3-K/AKT and SMAD2. Toxicol Lett 2017;265:53-60.

-30 Guan X: Cancer metastases: challenges and opportunities. Acta Pharm Sin B 2015;5:402-418.

-31 Bouris P, Skandalis SS, Piperigkou Z, Afratis N, Karamanou K, Aletras AJ, Moustakas A, Theocharis AD, Karamanos NK: Estrogen receptor alpha mediates epithelial to mesenchymal transition, expression of specific matrix effectors and functional properties of breast cancer cells. Matrix Biol 2015;43:42-60.

32 Lin TY, Hsu HY: Ling Zhi-8 reduces lung cancer mobility and metastasis through disruption of focal adhesion and induction of MDM2-mediated Slug degradation. Cancer Lett 2016;375:340-348.

-33 Niu X, Liu S, Jia L, Chen J: Role of MiR-3619-5p in beta-Catenin-Mediated Non-Small Cell Lung Cancer Growth and Invasion. Cell Physiol Biochem 2015;37:1527-1536.

34 Grooteclaes ML, Frisch SM: Evidence for a function of CtBP in epithelial gene regulation and anoikis. Oncogene 2000;19:3823-3828.

35 Lan L, Luo Y, Cui D, Shi BY, Deng W, Huo LL, Chen HL, Zhang GY, Deng LL: Epithelial-mesenchymal transition triggers cancer stem cell generation in human thyroid cancer cells. Int J Oncol 2013;43:113-120. 